\title{
Psychological Distress And Smoking Behaviors of Chinese College Students: Mediating Effects of The Dimensions of Learning Burnout
}

\author{
Xiong Li (D1173321980@qq.com) \\ Honghe Health Vocational College \\ Yuhua Tan \\ Yunnan Technology and Business University \\ Shanqing Li \\ Honghe Health Vocational College \\ Xiaoxin Wang \\ Honghe Vocational and Technical College
}

\section{Research Article}

Keywords: Psychological distress, Learning burnout, Smoking behavior, The theory of planned behavior

Posted Date: December 30th, 2021

DOI: https://doi.org/10.21203/rs.3.rs-1194303/v1

License: (c) (i) This work is licensed under a Creative Commons Attribution 4.0 International License. Read Full License 


\section{Abstract}

Objectives: Smokers or never smokers exposed to environmental tobacco use (ETS) are usually associated with various diseases and cancers. In order to better help college students prevent the tobacco use and thus preclude the incidences of avoidable diseases, this study explored the predictive power of different variables including demographic and psychological variables in relation to smoking behaviors.

Methods: Maslach Burnout Inventory-Student Survey (MBI-SS) and Kessler Psychological Distress Scale (K10) were used in this study.

Results: The multivariate logistic regression indicated that college students who were male (versus female, $O R=9.55)$, majoring in medicine and sports (versus nursing, $\mathrm{OR}_{\text {medicine }}=2.19, \mathrm{OR}_{\mathrm{sports}}=2.81$ ), born in the non-singleton family (versus singleton family, $\mathrm{OR}=0.63$ ) with higher family income (versus lower family income, $\mathrm{OR}=0.45$ ), surrounded with smoking friends (versus without smoking friends, $\mathrm{OR}=$ 0.18), were more vulnerable to smoke. In addition, combined with the theory of planned behavior (TPB), the dimensions of learning burnout had full mediation effects between psychological distress and smoking behavior.

Conclusions: Psychological distress can only indirectly affect smoking behavior via learning efficacy, cynicism and emotional exhaustion. Adjustment from different dimensions of learning burnout will help college students better prevent the tobacco use.

\section{Introduction}

Tobacco consumption is usually considered to be a double-edged sword which has negative impacts on public health and human longevity regardless of its positive effect on economic growth. Studies have found that as an unhealthy lifestyle, cigarette smoking could reduce people's life span by an average of seven years due to the complex compounds that cigarettes may contain, while most of which are carcinogens in human [1]. Those who got used to smoke in public not only undermined their own physical health, but also had the health of people around them endangered when they were smoking.

Cigarette smoking is presently found to be positively associated with nearly 40 diseases and causes of death for human, such as vascular diseases, oral diseases, chronic obstructive pulmonary disease (COPD), cancer of the lung, cancer of the liver, cancer of pancreas, cancer of renal pelvis and so on [2]. Smokers who have already formed the smoking habits are more likely to suffer from lung cancers than never smokers [3]. However, never smokers who expose themselves to environmental tobacco smoke (ETS) are also at high risks of various chronic diseases and cancers [4].

China is the country with the biggest tobacco consumption in the world [5]. The number of smokers in China had reached at least three hundred million in 2011 [6]. It was reported that the overall prevalence of tobacco smoking in China was up to $31.4 \%$ accounting for a quarter of the world's total smokers, while the total premature mortality resulting from cigarette smoking was $7.9 \%$ and the tobacco-related deaths 
were estimated to be 1 million annually in China $[7,8]$. These reported data may suggest that the tobacco control in China still needs improvement.

Generally speaking, the more the tobacco is consumed and the earlier the individual smokes, the greater the harm to the body as well as the higher the addiction will be. Compared with incidences of different cancers in China, the incidence of lung cancer ranked fourth in the 1970s and 1990s, whereas the incidence of lung cancer was constantly upgrading and had become the highest among all kinds of cancers since 2000 , the death rate from lung cancer had increased by $465 \%$ in the last 30 years accompanying with the remarkable increase of tobacco consumption [9].

The relation between physical health and cigarette smoking has been revealed in many studies, these studies have also indicated the current situation of smoking control in China is not optimistic, especially for Chinese youths and college students $[10,11]$. College students in China are more likely to smoke than others for some reasons. Firstly, Chinese culture is featured by Confucian and collectivism [12]. Given this kind of cultural influence, college students commonly prefer to imitate peers' smoking behaviors in order to socially integrate into the surroundings. Smoking has become the social label and peer bond for their relationships with others. Moran [13] has identified these young smokers as "social smokers" who show less intentions to quit smoking; Secondly, college students are usually interested in the things they have never come across. The curiosity on the new things and new experiences may lead them easily to smoke; Thirdly, college students in China have to face the fierce competition and learning stress at school. The competition and learning stress can increase the risks of smoking through which college students enable themselves to control and relieve their feelings of tension and stress [14].

In most cases, cigarette smoking is usually viewed as the method to mitigate one's anxiety and depression. For example, Hong et al. [15] applied different questionnaires to investigate 1068 fishermen, the impact of nicotine dependence upon negative emotions was revealed that nicotine dependence could partially counteract the negative effect of work stress upon depression and anxiety. With heavy work stress, fishermen often exhibited high levels of depression and anxiety. By the inhaling of cigarettes, the levels of depression and anxiety could be reduced for fishermen. Picciotto et al. [16] had also argued that the mitigation of negative emotions through cigarette smoking existed from the perspective of physiology, it was concluded that the physiological mechanism of cigarette smoking was due to the broad expression of nicotinic acetylcholine receptors (nAChRs), which further ascertained the positive effect of tobacco use upon emotional adjustment.

Despite the positive effect of tobacco use through which one can alleviate the levels of depression and anxiety, tobacco use is still harmful to the body. Moreover, excessive nicotine intake always produces biological adaption that could lead to the opposite effect and strengthen the smokers' existing depressions and anxieties, or even induce smoking-abstinence-related negative affect $[16,17]$. Therefore, due to plenty of hazards of smoking behaviors, effective tobacco prevention programs conducted in China especially for Chinese students are necessary. 


\section{The Present Study}

Although many studies have explored the impact of cigarette smoking upon emotion and cognition [1518], few of them have discussed whether negative emotions could contribute to the enhanced susceptibility of smoking behavior or not. In other words, appropriate cigarette smoking will certainly help regulate negative emotions while the likelihood of emotion-induced smoking behavior is not yet unveiled.

In order to verify the conviction that negative emotions could facilitate college students' smoking behaviors, learning burnout and psychological distress are taken into consideration in this article as the predictors of smoking behavior. Stoliker et al. [19] have pointed out that stressors such as learning burnout have become a common problem among students and something that they may encounter during their educational career. Learning burnout is mainly the internal threat giving rise to college students' mental health problems and unhealthy behavioral habits. Not only is learning burnout defined as a negative emotional state but also does it relate to psychological distress [19, 20]. The Chinese students who were haunted in the learning burnout usually failed the academic performance as well [21]. As a result, they would have to bear the enormous pressure from outside and inside, followed by the high levels of psychological distress and the increased inclinations of smoking.

Anyway, due to a variety of factors including learning burnout and mental illness might contribute to the inclination of cigarette smoking in theory, the influence of more possible variables would be explored in this article. This article aimed at building a multivariate logistic model to predict the smoking behaviors of college students based on a wide range of independent variables such as age, gender, majors, socioeconomic status (SES), family environment (FE), peer influence, learning burnout and psychological distress so that college students' smoking behaviors could be accurately predicted and intervened. Among all these variables, learning burnout and psychological distress were expected to be strongly associated with smoking behaviors. Therefore, we will explore the relationships among learning burnout, psychological distress and smoking behavior through empirical research, discover the possible mediation mechanism behind these variables and figure out the predictive power of different variables on smoking behavior. For these purposes, we further made three assumptions as follows.

\section{Hypothesis 1: Various Demographic Variables Would Predict Smoking Behavior}

Previous literature had confirmed that smoking behavior was significantly associated with age, gender, majors, SES, FE, peer influence [22, 23, 24]. Those people featured with high age (versus low age), male (versus female), art majors (versus science and engineering majors), high SES (versus low SES), disharmonious and broken FE (versus harmonious and complete FE) were more likely to smoke. Thus, age, gender, majors, SES and FE would be the demographic variables included in this article to verify the findings of previous studies. 
In addition, Wang et al. [24] had argued that friendship was also an inescapable factor that influence people's smoking decisions. Based on the data they collected, Wang et al. [24] compared people who had the increased number of smoking friends from 1989 to 1993 with those who had the decreased number of smoking friends during the same period, they found that people with the increased number of smoking friends were more likely to smoke. It seems not only does the quality of the friendship matter, but also what kind of friends people usually choose to make may to some extent determine the smoking behaviors. Thus, for purpose of verifying the conviction that friendship can affects college students' smoking behaviors, we sorted peer influence into the demographic variables and evaluated the predictive power of peer influence on smoking behavior by setting up an item like this: do you have any friends who usually smoke around you.

\section{Hypothesis 2: Learning Burnout and Its Different Dimensions Significantly Affect Smoking Behavior}

According to the theory of planned behavior (TPB) that was first come up with by Ajzen in 1988, perceived behavioral control (PBC), subjective norm (SN) and attitude, were constructed as the essential factors to predict individual's specific intention and behavior [25]. PBC refers to people's perception of the ease or difficulty of performing the behavior of interest. It was treated as the concept of self-efficacy which was first proposed by Bandura and his associates within a general framework [26]; Correlated with PBC in the TPB model, SN is a social factor that refers to the perceived social pressure to perform or not to perform the behavior and attitude refers to the degree to which a person has a favorable or unfavorable appraisal of the behavior [26]. It had been testified that all of these constructs in TPB model were available to predict a variety of health behaviors in many specific contexts [27]. In term of smoking behavior, TPB had already been confirmed to be the effective model to predict smoking behavior with all of its contents including PBC, SN and attitude significantly related to smoking behavior [28].

Although TPB was useful to predict smoking behavior, the effect sizes between TPB constructs and smoking behavior were relatively small [29]. As Mohiyeddini et al. [30] have argued, while TPB commonly shows high predictive power with respect to intention, it often falls short in the prediction of behavior, the key role in bridging the intention-behavior gap is emotion. In this sense, emotion should be added as the antecedent variable of smoking behavior in the TPB model.

Following this clue, we assumed that learning burnout as an emotional factor might relate to smoking behavior. Ling et al. [31] have concluded that learning burnout has three dimensions: emotional exhaustion, cynicism and learning efficacy. Emotional exhaustion refers to students' feelings of tiredness after learning. The individual who undergoes emotional exhaustion will excessively consume the inner cognitive resource and thus perform a sense of fatigue. He or she will eventually exhibit no extra energy and enthusiasm on learning and other behaviors such as smoking. So theoretically, it is predicted that emotional exhaustion would lead to the decreased inclination of smoking behavior.

Besides the emotional exhaustion, the remaining dimensions of learning burnout are similar to the whole constructs in TPB model (as shown in Figure 1). Since TPB model has been proven to be useful in the 
prediction of smoking behavior [29], it is thereby crucial to find out the theoretical connections between learning burnout and TPB model. However, these connections are made possible. Specifically, the three constructs in TPB model are found to correspond respectively to the two remaining dimensions in the measurement of learning burnout.

As one of the dimensions of learning burnout, learning efficacy shares the common origin with PBC though their directions and targets vary. That is, PBC in this case refers to the perceived ease or difficulty of smoking behavior, whereas learning efficacy points to the perceived ease or difficulty of learning. Due to the conceptual similarity between these two variables, we contend that learning efficacy could affect smoking behavior through PBC for people's disposition of transferring similar knowledge from one area to another [32].

Cynicism, as another dimension of learning burnout, resembles the attitude and SN in TPB model. Cynicism by definition contains two meanings: one is that it represents the preference of learning or to what extent students hate to study, which can be regarded as the construct of attitude in TPB model; The other meaning that cynicism embodies is the students' judgement and beliefs about the importance of learning, including their reflections on what kind of social influence learning would produce and what kind of social pressure they would have to face once the poor academic performances happen, thus, SN which represents the perceived social pressure to quit smoking and cynicism which embodies social pressure to keep learning are the intertwined variables with conceptual similarity. From this point of view, cynicism can be regarded as $\mathrm{SN}$ and attitude toward learning behavior, with its highly probable influence on smoking behavior through $\mathrm{SN}$ and attitude toward smoking by transfer learning.

In sum, cynicism and learning efficacy are two dimensions of learning burnout corresponding to $\mathrm{SN}$, attitude and PBC in TPB model. Meanwhile, according to the viewpoint that people's knowledge gained from specific cases can then be transferred to the novel situations naturally [32], it is reasonable to predict that cynicism and learning efficacy can affect smoking behavior through SN, attitude and PBC. Consistent with the above-mentioned inferences, we hypothesize that learning burnout and all its different dimensions could affect smoking behavior.

\section{Hypothesis 3:Learning Burnout and its Dimensions Mediate the Relation Between Psychological Distress and Smoking Behavior}

The self-medication hypothesis postulates that individuals always turn to smoking to alleviate the symptoms therefore suggests that symptoms of depression and anxiety may lead to smoking [33]. Individuals with mental illness and symptoms of depression and anxiety tended to start smoking at an earlier age, smoke more heavily, and were more addicted to cigarettes than the general population [33]. Therefore, mental illness may be an important variable relative with tobacco use, the link has often been taken to reflect a causal relationship, with poor mental health predisposing to regular tobacco use [34]. 
This phenomenon was specifically found in White individuals and pregnant women instead of Black or Hispanic individuals $[28,35]$.

Given the relation between psychological distress and smoking behavior, there is a plausible prospect that psychological distress which synthesizes elements of negative emotions and mental illness is capable of affecting the inclination of smoking behavior.

Although learning burnout and psychological distress are both likely to influence smoking behavior, in TPB model, learning burnout and all its dimensions are closer to smoking behavior than psychological distress, so we hypothesize that learning burnout and its dimensions could mediate the relation between psychological distress and smoking behavior for the same purpose of revealing the concealed mechanism behind emotion-induced smoking behaviors for college students.

\section{Methods}

\section{Participants}

A cross sectional study was conducted. The questionnaires were administered to two colleges by convenient sampling. There was a total of 1449 college students participating in the study and the data were collected after completion by the college students. After carefully screening the missing or inconsistent responses from the collected data (e.g., the definition of inconsistent responses: the item 6: " have you always smoked" with students' answers: "no" contradicted the item 7: " how frequently have you smoked" with the answers: " $i$ have smoked at least 100 times in a month"), 1340 pieces of data were left for further analysis. Correspondingly, the participants in the analyzed data were aged 18.83 on average with 1.55 standard deviation.

\section{Procedures}

The study has been approved by the academic committees of Honghe Health Vocational college and Yunnan Technology and Business University since it was planned. The academic committee of Yunnan Technology and Business University approved the research protocol and conducted study oversight and monitoring. The academic committee of Honghe Health Vocational college signed the ethical approval after the scrutiny of the measuring tools and the design of study process.

Before measuring, a sample of 1449 college students from different colleges volunteered to participate in the study with oral agreement and received two course credits as encouragement at the end of the study. The anonymity and purposes of the measurements were well known by the participants. All the participants gave informed consent to the measurements and were required to be genuine to respond. Then the measurements were carried out at different times and places so as to strictly control the common method deviation [36]. The personal information of the participants (e.g., name, phone number, address) was not collected in this study for subjecting to the ethical standards.

\section{Measures}




\section{Learning burnout}

Learning burnout was measured with Maslach Burnout Inventory-Student Survey (MBI-SS) [37]. This questionnaire stemmed from Maslach Burnout Inventory (MBI) which was originally used for the measurement of job burnout, previous studies found it not only applicable on workplace but also available for students, so MBI was revised and enriched to become the MBI-SS [38]. It was reported that the Cronbach's alpha of MBI-SS was $0.84-0.90$, test-retest reliability of MBI-SS was $0.67-0.89$, concurrent validity for its various dimensions were obtained $0.74,0.68$ and 0.50 respectively [39].

In the present study, MBI-SS as a 7-points frequency rating scale comprised of 15 items with each item ranged from 0 (Never) to 6 (always) was used. MBI-SS has three dimensions such as emotional exhaustion, cynicism and learning efficacy, the Cronbach's alpha for each of its dimensions in this study was $0.82,0.82$ and 0.85 respectively, the Cronbach's alpha of the total scale was 0.86 . Learning burnout was represented by the summation of each of its dimensions with reverse score of learning efficacy notified.

\section{Psychological distress}

The Kessler Psychological Distress Scale (K10) was constructed based on Item Response Theory (IRT) which was a validated 10-item scale measuring the non-specific symptom of psychological distress [40]. Each item in the K10 has 5 points ranged from 1 (None of the time) to 5 (Almost all of the time) representing the frequency rating of each item over the four weeks [41]. With higher score meaning worse mental health, the K10 in Chinese version was applied in this study with its acceptable reliability and validity ever reported by Wen et al. [42] (e.g., Kappa is 0.70 , split-half reliability is 0.71 and Cronbach's alpha is 0.80 ). Anyway, based on this study, the internal consistency of the K10 in Chinese version was 0.93 .

\section{Statistical analysis}

Descriptive analysis, chi-square test and logistic regression model were adopted. The alpha value was set at 0.05. Data were analyzed using SPSS 18 (IBM Corp., Armonk, NY, United States).

Associations between demographic variables and smoking behavior were analyzed by chi-square test. Those demographic variables unrelated to smoking behavior, in this case which did not pass the chisquare test, would not enter into the logistic model on the next step. Subsequently, the set of independent variables in the multivariate logistic model would include the remaining demographic variables, psychological distress, learning burnout and all its dimensions whereas the dependent variable in the logistic model was occupied by the occurrence of smoking behavior. Finally, in order to investigate the mediating effects of learning burnout and its various dimensions, we use the mediation method proposed by lacobucci D. [43] which is unlike the traditional mediation procedures because of the dichotomous dependent variable. Mediation method in this study complied with the following procedures:

(1) Fit $X$ and $Y$ via a logistic model: 
$\mathrm{Y}=e 1+c X$

The intercept $\mathrm{e} 1$ and the slope $c$ were produced along with their standard errors (SE).

(2) Fit $\mathrm{X}$ and $\mathrm{M}$ via a linear regression model:

$$
M=e 2+a X .
$$

The slope $a$ was indicated along with the corresponded SE. Collected the parameter estimate $a$ and its standard error, $s_{a}$.

(3) Fit $X, M$ and $Y$ via a regression model:

$$
Y=e 3+c^{\prime} X+b M
$$

Collected the parameter estimate $b$ and its standard error, $s_{b}$.

(4) Using the collected parameter estimates and calculating the mediation effect by the following formula:

$$
Z_{\text {Mediation }}=\frac{\frac{a}{s_{a}} \times \frac{b}{s_{b}}}{\sqrt{\left(\frac{a}{s_{a}}\right)^{2}+\left(\frac{b}{s_{b}}\right)^{2}+1}} .
$$

Test $Z_{\text {Mediation }}$ against a standard normal distribution, the mediation effect is found if $Z_{\text {Mediation }}$ exceeds |1.96| for a 2-tailed test with $a=0.05$ [42]..

\section{Results}

\section{Descriptive analysis and chi-square test}

A demographic survey including different variables were practiced. Descriptive analysis and chi-square test were used to explore the performances of different variables upon smoking behavior. 
Table 1

Demographic characteristics of present study cohort of college students

\begin{tabular}{|c|c|c|c|c|}
\hline \multirow[t]{2}{*}{ Variables } & \multirow{2}{*}{$\begin{array}{l}\text { Frequency } \\
(n=1340)\end{array}$} & \multicolumn{3}{|c|}{ Smoking behavior } \\
\hline & & $\begin{array}{l}\text { Never smoke } \\
(n=1037)\end{array}$ & $\begin{array}{l}\text { Have ever smoked } \\
(n=303)\end{array}$ & $x^{2}$ \\
\hline Gender & & & & $366.56 * \star$ \\
\hline Male & $37.1 \%$ & 243 & 254 & \\
\hline Female & $62.9 \%$ & 794 & 49 & \\
\hline Majors & & & & $201.24^{\star \star}$ \\
\hline Pedagogy & $29.2 \%$ & 356 & 35 & \\
\hline Medicine & $23.9 \%$ & 243 & 77 & \\
\hline Literature and Art & $3.2 \%$ & 39 & 4 & \\
\hline Engineering & $1.3 \%$ & 11 & 7 & \\
\hline Sports & $23.4 \%$ & 159 & 155 & \\
\hline Nursing & $19.0 \%$ & 229 & 25 & \\
\hline Ethnicity & & & & $10.45^{\star}$ \\
\hline Han & $55.8 \%$ & 580 & 168 & \\
\hline Yi & $19.4 \%$ & 206 & 54 & \\
\hline Hani & $4.3 \%$ & 35 & 23 & \\
\hline Other minority & $20.4 \%$ & 216 & 58 & \\
\hline Socioeconomic status(SES) & & & & $11.43^{\star \star}$ \\
\hline$<3000 y u a n$ & $31.6 \%$ & 343 & 80 & \\
\hline 3000-10000yuan & $53.8 \%$ & 559 & 162 & \\
\hline > 10000yuan & $14.6 \%$ & 135 & 61 & \\
\hline \multicolumn{5}{|l|}{ Family environment(FE) } \\
\hline Single-parent household & $11.0 \%$ & 115 & 33 & 0.01 \\
\hline Two-parent household & $89.0 \%$ & 922 & 270 & \\
\hline Singleton family & $17.0 \%$ & 161 & 67 & $7.21^{\star \star}$ \\
\hline Non-singleton family & $83.0 \%$ & 876 & 236 & \\
\hline
\end{tabular}




\begin{tabular}{|c|c|c|c|c|}
\hline \multirow[t]{2}{*}{ Variables } & \multirow{2}{*}{$\begin{array}{l}\text { Frequency } \\
(n=1340)\end{array}$} & \multicolumn{3}{|c|}{ Smoking behavior } \\
\hline & & $\begin{array}{l}\text { Never smoke } \\
(n=1037)\end{array}$ & $\begin{array}{l}\text { Have ever smoked } \\
(n=303)\end{array}$ & $\chi^{2}$ \\
\hline Domicile place & & & & 3.80 \\
\hline Countryside & $82.6 \%$ & 868 & 239 & \\
\hline Town & $17.4 \%$ & 169 & 64 & \\
\hline Peer influence & & & & $105.34^{\star *}$ \\
\hline With smoking friends & $70.4 \%$ & 658 & 285 & \\
\hline Without smoking friends & $29.6 \%$ & 379 & 18 & \\
\hline
\end{tabular}

As shown in Table 1, gender, majors, peer influence, SES and singleton family were significantly correlated with smoking behavior.

However, there were two variables not significantly relative with smoking behavior (e.g., domicile place and single-parent family). It seemed that no matter whether people came from countryside or town, the likelihood of occurrences of cigarette smoking did not vary. The phenomenon was the same as whether people belonged to single-parent families or not. The result might support the viewpoint that with the socialization proceeding, the influence power of original family on individual's behavior and capability would attenuate [36].

\section{Logistic Regression Model}

Based on the analysis of chi-square test, gender, majors, SES, FE, and peer influence were included in the logistic regression model (LRM). The regression dummy variables in LRM were respectively gender ( male $=1$, female $=2$ ), majors (pedagogy $=1$, medicine $=2$, literature and art $=3$, engineering $=4$, sports $=5$, nursing = 6), SES (<3000yuan = 1,3000-10000yuan $=2,>10000$ yuan $=3$ ), FE (singleton family $=1$, nonsingleton family $=2$ ), peer influence ( with smoking friends $=1$, without smoking friends $=0$ ). 
Table 2

Demographic Variables in Prediction of Smoking Behavior

\begin{tabular}{|lccccc|}
\hline Variables & B & S.E. & $\boldsymbol{p}$ & OR & 95\%CI \\
\hline Age & -0.05 & 0.08 & 0.49 & 0.95 & $0.81-1.11$ \\
\hline Gender(control group = female) & 2.26 & 0.19 & 0.00 & 9.55 & $6.48-14.06$ \\
\hline majors(control group = nursing) & & & & & \\
\hline Pedagogy & 0.01 & 0.37 & 0.98 & 1.01 & $0.49-2.07$ \\
\hline Medicine & 0.78 & 0.29 & 0.00 & 2.19 & $1.25-3.85$ \\
\hline Literature and Art & 0.02 & 0.68 & 0.97 & 1.02 & $0.26-3.86$ \\
\hline Engineering & 0.16 & 0.58 & 0.78 & 1.17 & $0.37-3.64$ \\
\hline Sports & 1.03 & 0.33 & 0.00 & 2.81 & $0.46-5.41$ \\
\hline Ethnicity (control group = minority) & & & & & \\
\hline Han & -0.01 & 0.16 & 0.97 & 0.99 & $0.72-1.37$ \\
\hline SES(control group = >10000yuan) & & & & & \\
\hline < 3000yuan & -0.80 & 0.25 & 0.00 & 0.45 & $0.27-0.73$ \\
\hline 3000-10000yuan & -0.35 & 0.23 & 0.12 & 0.70 & $0.44-1.10$ \\
\hline FE (control group = non-singleton family) & & & & & \\
\hline Singleton family & -0.46 & 0.21 & 0.03 & 0.63 & $0.41-0.96$ \\
\hline Peer influence(control group = with smoking friends) & & & & & \\
\hline Without smoking friends & -1.74 & 0.27 & 0.00 & 0.18 & $0.10-0.29$ \\
\hline Encoding of smoking behavior: 1 = Have ever smoked, $0=$ Never smoke. & & & \\
\hline
\end{tabular}

As for Han was the largest Chinese ethnicity, we renewed the ethnicity variable by encoding Yi, Hani and other minority into one category with Han as the other main category. As shown in Table 2, hypothesis 1 was partially supported by the analytic result that many demographic variables were significantly predictable of smoking behavior except age and ethnicity. That is, just like ethnicity, age has no impact on the likelihood of smoking behavior. It was inconsistent with previous studies probably because of the limited age scope of the involved participants and the unbalanced quantity of each ethnicity in this study.

However, the main findings in previous studies were testified to be valid again. College students who were male $(O R=9.55)$, majoring in medicine and sports $\left(O R_{\text {medicine }}=2.19, \mathrm{OR}_{\text {sports }}=2.81\right)$, born in nonsingleton family $(O R=0.63)$, with higher family income $(O R=0.45)$, surrounded with smoking friends $(\mathrm{OR}=0.18)$, were more likely to smoke than female students who were majoring in nursing, born in singleton family with lower family income, surrounded without smoking friends. 
Psychological variables including learning burnout and psychological distress seemed not capable of affecting the occurrence of smoking behavior due to the analytic result of logistic model as shown in Table 3. However, learning efficacy, cynicism and emotional exhaustion could significantly influence smoking behavior as were covariant with the total score of learning burnout and psychological distress. This result partially supported hypothesis 2 , in which the assumption that all the dimensions of learning burnout could affected smoking behavior as TPB model theoretically implied was proven.

Table 3

Psychological Variables in Prediction of Smoking Behavior

\begin{tabular}{|llllll|}
\hline Factors & B & S.E. & $\boldsymbol{p}$ & OR & 95\%Cl \\
\hline Learning efficacy & -0.02 & 0.01 & 0.02 & 0.98 & $0.96-0.99$ \\
\hline Cynicism & 0.06 & 0.02 & 0.00 & 1.06 & $1.02-1.10$ \\
\hline Emotional exhaustion & -0.05 & 0.02 & 0.00 & 0.95 & $0.92-0.98$ \\
\hline Learning burnout & 0.01 & 0.01 & 0.10 & 1.01 & $0.99-1.02$ \\
\hline Psychological distress & -0.01 & 0.01 & 0.77 & 0.997 & $0.97-1.02$ \\
\hline
\end{tabular}

\section{Mediation Model}

According to the analysis above, learning burnout was definitely not able to mediate the relation between psychological distress and smoking behavior. What's more, psychological distress was also incapable of affecting the inclination of smoking behavior for college students. So in order to delve into the reason for the insignificant path between psychological distress and smoking behavior, we divided the continuous variable psychological distress into four categories by the cut-off points proposed by Wen, et al. [42]. That is, students scoring 10-15 were distributed into the least distress group with the best mental health, scoring 16-21 into less distress group, scoring 22-29 into more distress group and scoring 30-50 into most distress group with the worst mental health.

As shown in Figure 2, the descriptive result indicated that college students who fell into most distress group were most likely to smoke (32.6\%) with the minimal possibility to become never smokers (67.4\%), whereas other groups especially for more distress group were least possible to smoke with the greater possibility to become never smokers on the contrary (e.g., there were $17.89 \%$ college students in more distress group belonging to smokers and $82.1 \%$ of them belonging to never smokers). The smoking behavior was proven to be correlated with different categories of psychological distress, the chi-square test $\chi^{2}$ between these two variables was 11.99 with statistical significance $(p<0.05)$, but the conclusion that psychological distress could not directly affect smoking behavior remained unchanged.

As mentioned before, psychological distress was correlated with smoking behavior. However, psychological distress could not affect smoking behavior directly. It was possible that there might be a masking effect or full mediation effect leading to the insignificant path between psychological distress 
and smoking behavior. Thus, further analysis to reveal the invisible mechanism behind these two variables is necessary. The mediation method for categorical variable was then carried out.

As shown in Figure 3, psychological distress can significantly influence smoking behavior only through the dimensions of learning burnout. The $Z$ scores for mediation effects of learning efficacy, cynicism and emotional exhaustion were $2.23,2.98$ and -3.09 respectively, the values of mediation effects all exceeded |1.96| which meant the mediation effects of the dimensions of learning burnout were significant statistically, thus hypothesis 3 was partially supported. A full mediation effect mechanism was revealed in this study that psychological distress could not directly affect the likelihood of smoking behavior, but it could indirectly exert impacts on smoking behavior via learning efficacy, cynicism and emotional exhaustion, the dimensions of learning burnout were found to play an important role on the relationship between psychological distress and smoking behavior.

\section{Discussion}

In the present study, we built up a logistic model including all kinds of demographic variables in the prediction of smoking behavior. It was found that college students who were male, majoring in medicine and sports, born in the non-singleton family, with higher family income, surrounded with smoking friends, were more likely to smoke than female students who were nursing major, born in the singleton family, with lower family income, surrounded without smoking friends. Many findings in previous literature have been testified to be valid in this article. Moreover, we examined the relationships between psychological variables and smoking behavior. The intricate relationships among these two kinds of variables were partially revealed. The findings in this article may help the college administrators and college teachers instruct their students to better prevent the tobacco use, it's also conducive to establish the warning system in which college students who are vulnerable to smoke will be concentrated on.

\section{The Dimensions Of Learning Burnout Significantly Affect Smoking Behavior}

As for Chinese college students, learning is the main task undertaking great expectations from their parents. Almost every Chinese parents would wish their children have good performances on learning. However, the ones who failed the academic achievement and suffered from learning burnout would undoubtedly bear the enormous stress from outside or inside, as a result, relying on smoking to release the feelings of stress and anxiety was more likely to happen on these students. This study has proven that though learning burnout can not affect smoking behavior due to the broad implication it refers to, its concrete and specific dimensions can significantly affect smoking behavior in somehow.

It has been found in this article that college students with higher learning efficacy and higher emotional exhaustion, in addition to lower cynicism on learning, are hardly to smoke. To understand this, TPB model can be combined with. Learning efficacy is similar to PBC in the constructs of TPB model, it represents 
the senses of behavioral control for students. Those students with higher learning efficacy will be more confident of controlling their learning behaviors, this experience can then be transferred to other scenarios such as smoking, thus they will be more willing and capable of resisting the attempts to smoke especially when they recognize the harmfulness of smoking; Emotional exhaustion as another special construct in learning burnout, it represents the feeling of tiredness and fatigue after learning. Accompanying with higher emotional exhaustion, individuals may invest too much time and energy into the school learning, their great passion and enthusiasm were exhausted by school learning so that they had less cognitive resources to spare on smoking, thus higher emotional exhaustion usually indicates less probability of smoking behavior; Cynicism represents students' preferences on learning. With higher levels of cynicism, students are more prone to immerse themselves by the senses of social pressure on learning, thus smoking gradually becomes a visible option for them to relieve the stress, which eventually increase their vulnerabilities of smoking behaviors.

\section{The Full Mediation Effects Of Dimensions Of Learning Burnout}

Although psychological distress can not affect smoking behavior directly, it can indirectly influence smoking behavior through the full mediation mechanism of the dimensions of learning burnout. For example, students with higher distress levels often exhibit lower learning efficacy, and then students with lower learning efficacy are easier to become smokers as a result of inability to inhibit the unhealthy behavior; Additionally, psychological distress can also enhance students' cynicism toward learning, students immersing in higher levels of psychological distress are easier to negatively think of learning and detest learning, therefore they are more vulnerable to smoke because smoking has gradually become one of the visible options for them to relieve the stress with more and more investments of time and efforts probably expended on other unhealthy activities and behaviors except learning. However, it is noteworthy that psychological distress can reduce the likelihood of smoking behavior in reverse through emotional exhaustion. Students with higher levels of distress are easier to deplete their patience on learning, it will cost them much time and energy to experience and express sadness, sorrow, anxiety, depression and so on when they were learning, which contribute to their increased levels of emotional exhaustion, and then it is possible that they have no energy or enthusiasm to spare on smoking.

In conclusion, in order to prevent college students from the tobacco use, the importance of learning should be emphasized. College administrators and college teachers can better help their students resist the attempts to smoke indirectly by adjusting college students' emotional and cognitive reactions toward learning. For example, by lowering the difficulty of curriculum, reshaping students' internal attribution styles and encouraging students to be more confident of their studies, college students' learning efficacy will be enhanced and their cynicism on learning will be reduced. Besides, by completing educational reform to absorb affective education, college administrators and college teachers will be able to regulate college students' emotional reactions on learning, strengthen their motivations to learn and help college students better devote themselves into the learning with great passion and enthusiasm, the students with 
the abundant emotional experience on learning will be more obsessed with the exploration of the ocean of knowledge, and also be more possibly haunted by the feelings of tiredness after the duration of learning, as a result, the increased emotional exhaustion will be good for smoking prevention.

Anyway, adhering to the specific measures and the practical principles this article has implied, the learning efficacy of college students will be improved as well as their increased emotional exhaustion, along with the decrease of cynicism, college students can be less willing to smoke in contrast to more willingness to concentrate on school learning. In such case, the opportunities to smoke and the environmental factors should also be considered for future studies.

\section{Conclusion}

This article suggests that although proper tobacco consumption enables college students to socially consolidate their friendships with other people and relieve their feelings of tension and stress, it still puts college students' physical health in great danger considering their strong abilities to accept new things and the growing addiction smoking behaviors may result in. Thus, effective tobacco preventive programs and warning system are essential to set up for colleges students worldwide.

It has been found that there are 303 college students in this study addicted to smoke, accounting for $22.61 \%$ of the total sample. College students who were male, majoring in medicine and sports, born in non-singleton family with higher family income, surrounded with smoking friends, were more vulnerable to smoke. The warning system can be used once the bottom line is triggered by the criteria that the students who match the mentioned conditions should be concentrated on and be taken measures to prevent them from tobacco use in advance.

In addition, the article has figured out the specific measures to prevent college students from tobacco use, i.g., due to the full mediation effects of the dimensions of learning burnout, college teachers and administrators can actively enhance college students' learning efficacy and emotional exhaustion, avoid the increased cynicism toward learning. Through this way, college students will be less likely to smoke.

\section{Declarations}

All procedures performed in the study were in accordance with the ethical standards of academic committees of Honghe Health Vocational college and Yunnan Technology and Business University.

\section{Acknowledgements}

We thank the students who participated in the current study and also thank for the assessment support from the academic committee of Honghe Health Vocational college.

\section{Data Availability}


The datasets generated during and/or analyzed during the current study are available from the corresponding author on reasonable request.

\section{Ethics approval and consent to participate}

The study was conducted according to the principles in the declaration of Helsinki. The academic committees of Honghe Health Vocational college and Yunnan Technology and Business University approved the research protocol. Informed consent was obtained from each participant prior to data collection. They were also informed of the purpose of the study, their voluntary participation, and that there were no foreseeable risks or harms in the study.

\section{Consent to Participate}

Informed consent was obtained from all of individual participants included in the study.

\section{Conflict of Interest}

The authors declare that they have no conflict of interest .

\section{References}

1. Bernhard D, et al. Cigarette smoke-an aging accelerator?. Experimental Gerontology. 2007; 42(3):160-165.

2. Vellappally S, et al. Smoking Related Systemic and Oral Diseases. Acta Medica. 2007; 50(3):161-166.

3. Watanabe M. Smoking: additional burden on aging and death. Genes \& Environment.2016; 38(1):3.

4. Barbara E, et al. Smoking-related disease risk, area deprivation and health behaviours. Journal of Public Health. 2013; 36(1): 72-80.

5. Jiang $Y$, et al. Chinese Physicians and Their Smoking Knowledge, Attitudes, and Practices. American Journal of Preventive Medicine. 2007; 33(1):15-22.

6. Yuan J, et al. Epidemic and control on tobacco in China. Chinese Journal of Epidemiology. 2011; 32(012):1181-1187.

7. Schroergunther M, et al. Primary tobacco prevention in China--a systematic review. Asian Pacific Journal of Cancer Prevention. 2011; 12(11):2973-2980.

8. Gruder CL, et al. Tobacco Smoking, Quitting, and Relapsing Among Adult Males in Mainland China: The China Seven Cities Study. Nicotine \& Tobacco Research. 2012; 15(1):223-230.

9. Lin $X$, Jie Y. China tobacco related disease and economic burden. Chinese journal of health education. 2008; 24(9):723-725.

10. Johnson CA, et al. Tobacco use among youth and adults in Mainland China: The China Seven Cities Study. Public Health. 2006; 120(12):1156-1169. 
11. Shuaijun G, et al. Cluster analysis of smoking, alcohol drinking and other health risk behaviors in undergraduate student. Journal of Peking University. 2013; (03):382-386.

12. Wang ZG.Comparison of Chinese and Western Value from four Kinds of Angles. Journal of Lianyungang Teachers College. 2009; 26(3):45-48.

13. Moran S. Social Smoking Among US College Students. PEDIATRICS. 2004; 114(4): 1028-1034.

14. Rongxian W, et al. Cigarette smoking and tobacco attitudes among undergraduate students in China. The Journal of Medical Theory and Practice. 2002: (05):42-44.

15. Hong JJ, et al. Work Stress and Depressive Symptoms in Fishermen With a Smoking Habit: A Mediator Role of Nicotine Dependence and Possible Moderator Role of Expressive Suppression and Cognitive Reappraisal. Frontiers in Psychology. 2018; 9:386.

16. Picciotto MR, et al. Effect of nicotine and nicotinic receptors on anxiety and depression. Neuroreport. 2002; 13(9):1097.

17. David GG, et al. Effects of smoking abstinence on mood and craving in men: influences of negativeaffect-related personality traits, habitual nicotine intake and repeated measurements. Personality and Individual Differences. 1998; 25(3):399-423.

18. Nikcevic AV, Spada MM. Metacognitions about smoking: a preliminary investigation. Clinical Psychology \& Psychotherapy. 2010; 17(6):536-542.

19. Stoliker BE, Lafreniere KD. The Influence of Perceived Stress, Loneliness, and Learning Burnout on University Students' Educational Experience. College Student Journal. 2015; 49(1):146-160.

20. Maoping L. The Correlation of College Students' Mental health and Learning Burnout. Journal of Lishui University. 2015; 1:113-118.

21. Dan $P$, et al. Research on coping style improved scores through ameliorating learning burnout. China Journal of Health Psychology. 2020; 28 (4):604-607.

22. Wei $L$, et al. Analysis on epidemic status and influencing factors of smoking, drinking and drug addiction among adolescents in Liuzhou. Journal of Medical Pest Control. 2012; 28(8):846-850.

23. Yong $X$, Jing LY. Psychosocial Risk Factors and Family Psyche Environment of Smoking Adolescents. Chinese Mental Health Journal. 2004; 18(7):504-506.

24. Wang MQ, et al. Smoking acquisition: peer influence and self-selection. Psychological Reports. 2000; 86(3 Pt 2): 1241-6.

25. Özer G, Yilmaz E. Comparison of the Theory of Reasoned Action and the Theory of Planned Behavior: An Application on Accountants' Information Technology Usage. Social Science Electronic Publishing. 2011; 5(1):50.

26. Ajzen, I. The theory of planned behavior. Organizational Behavior \& Human Decision Processes. 1991; 50(2):179-211.

27. Kwan WYM, et al. Predicting Physical Activity of First-Year University Students: An Application of the Theory of Planned Behavior. Journal of American College Health. 2009; 58(1):45-52. 
28. Marc TK, et al. Psychological distress and smoking behavior: the nature of the relation differs by race/ethnicity. Nicotine \& Tobacco Research. 2010; 13(2):113-119.

29. Gabriela T, Juan AM. Theory of planned behavior and smoking: meta-analysis and SEM model. Substance Abuse \& Rehabilitation. 2010; 1:23-33.

30. Mohiyeddini $C$, et al. The role of emotion in bridging the intention-behaviour gap: The case of sports participation. Psychology of Sport \& Exercise. 2009; 10(2):226-234.

31. Ling $L$, et al. An investigation about learning burnout in medical college students and its influencing factors. International Journal of Nursing Sciences. 2014; 1(1):117-120.

32. Gentner $D$, et al. Learning and transfer: a general role for analogical encoding. Journal of Educational Psychology. 2003; 95(2):393-408.

33. Meg F, et al. The Association of Cigarette Smoking With Depression and Anxiety: A Systematic Review. Nicotine \& Tobacco Research. 2017; (1):3-13.

34. Patton GC, et al. Is smoking associated with depression and anxiety in teenagers? American Journal of Public Health. 1996; 86(2):225-230.

35. Goodwin RD, et al. Serious psychological distress and smoking during pregnancy in the united states: 2008-2014. Nicotine \& Tobacco Research. 2017; 19(5):605-641.

36. Xiong L, et al. An Exploration into the Influence of Higher Vocational Students' Psychological Capital on Their Employability. SHS Web of Conferences. 2021; 96:02007.

37. Qiao H, Schaufeli WB. The factorial validity of the Maslach Burnout Inventory-Student Survey in China. Psychological Reports. 2009; 105(2):394-408.

38. Zhang $Y$, et al. The reliability and validity of MBI-SS and academic characteristics affecting burnout. Chinese Journal of Clinical Psychology. 2005; 13(4):383-385.

39. Rostami Z, et al. The Psychometric Characteristics of Maslach Burnout Inventory Student Survey: A Study Students of Isfahan University. Zahedan journal of research in medical sciences. 2014; 16(9):55-58.

40. Kessler RC, et al. Short screening scales to monitor population prevalences and trends in non-specific psychological distress. Psychological Medicine. 2002; 32:956-959.

41. Xuan W, et al. Mental Health and its Influence Factors among the Primary Tuberculosis Patients. Chinese Journal of Health Statistics. 2015; 32(2):194-196.

42. Wen GZ, et al. Application of Kessler 10 Rating Scale in Study on Relationship between Accidental Injury and Mental Health Status. Chinese Mental Health Journal. 2009; 32(3):175-191.

43. Iacobucci D. Mediation analysis and categorical variables: the final frontier. Journal of Consumer Psychology. 2012; 22:582-594.

\section{Figures}




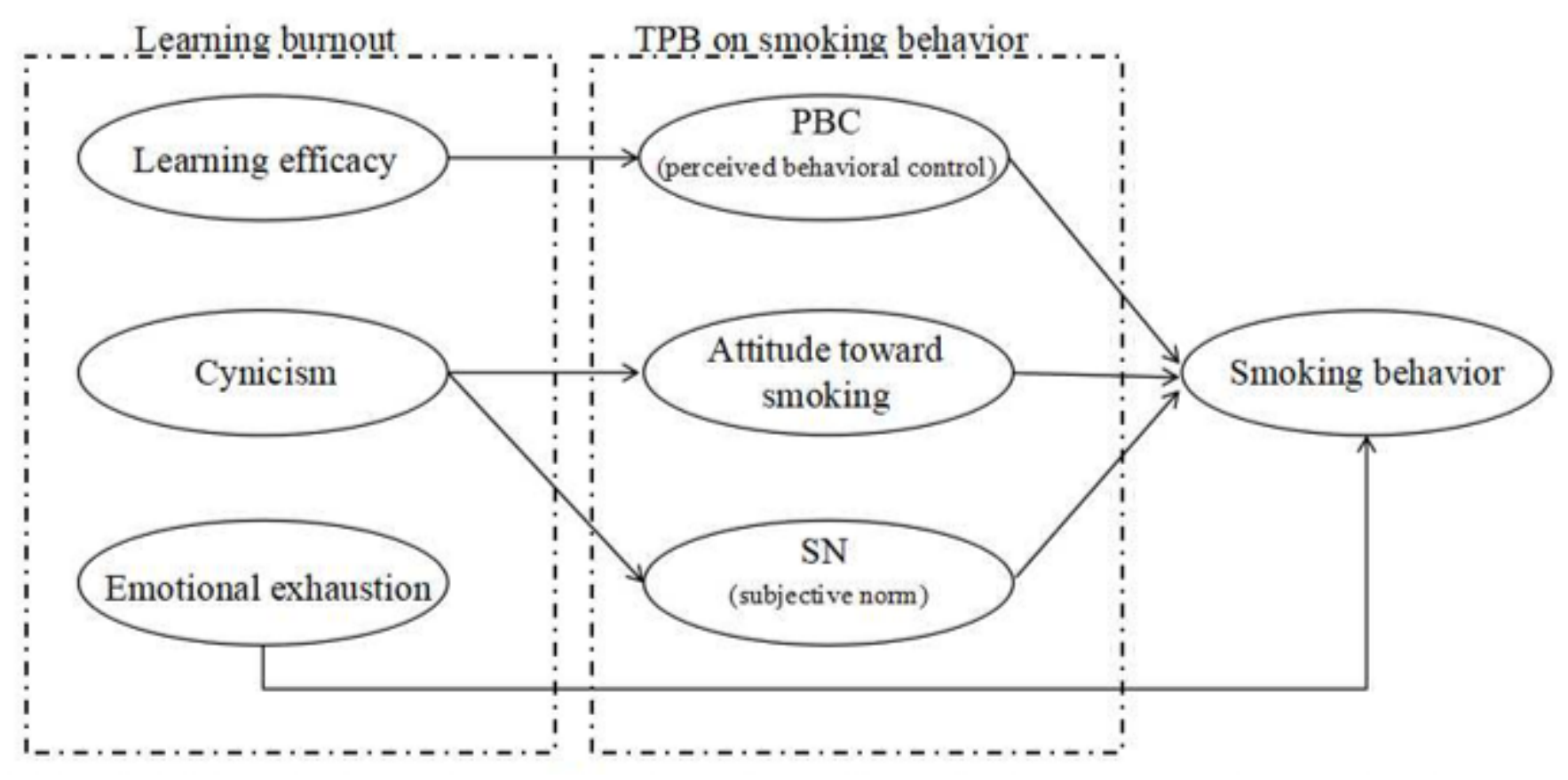

\section{Figure 1}

The relation between the dimensions of learning burnout and the constructs of TPB model toward smoking behavior.

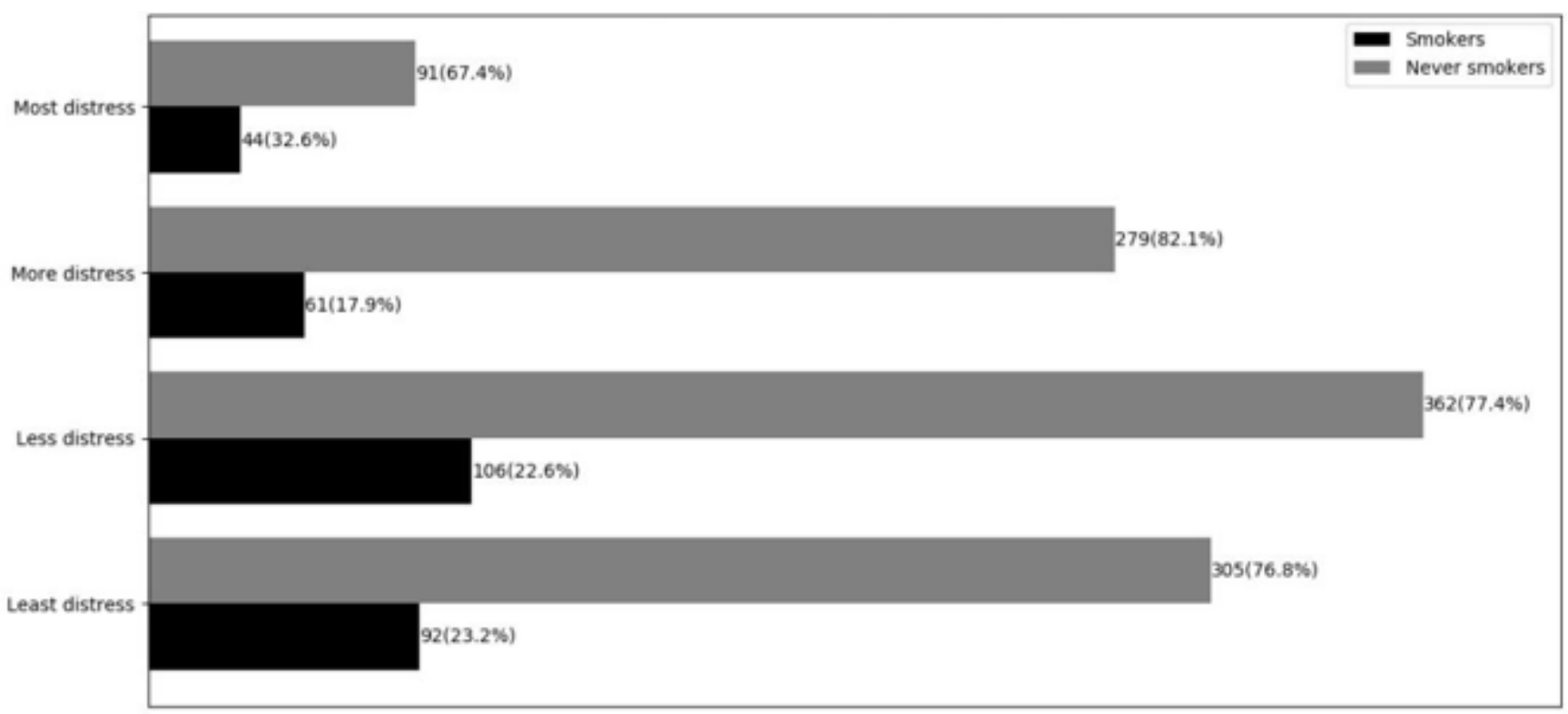

\section{Figure 2}

Frequency of Different Types of Psychological Distress 


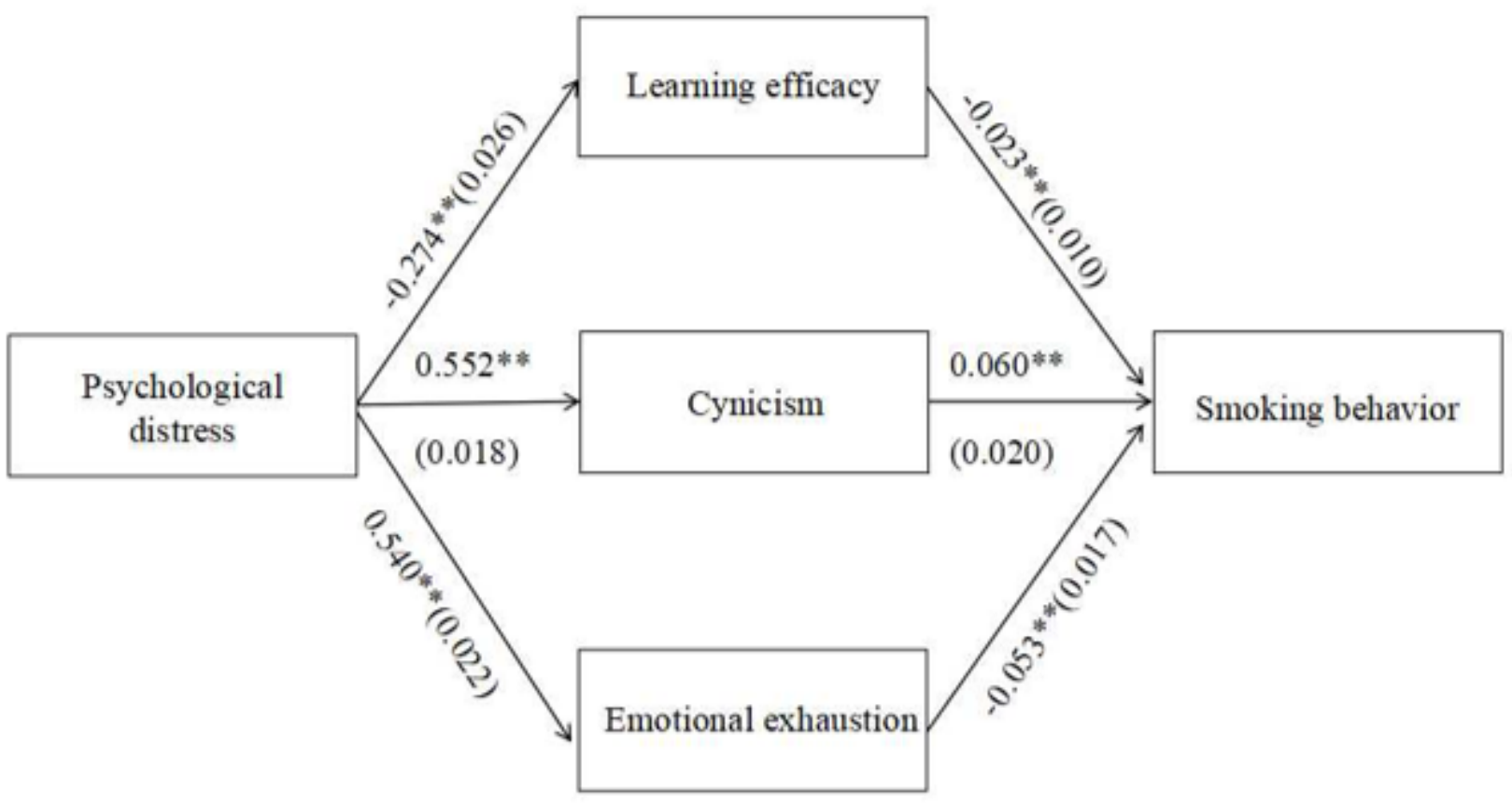

\section{Figure 3}

The Influence Mechanism of Psychological Distress upon Smoking Behavior. 